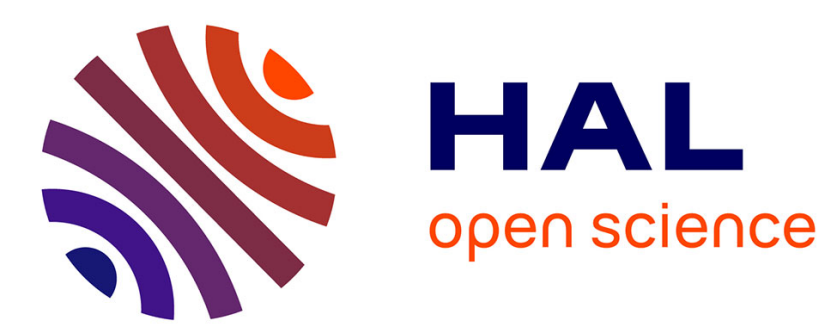

\title{
Telegraph noise due to domain wall motion driven by spin current in perpendicular magnetized nanopillars
}

J Cucchiara, Y Henry, D Ravelosona, D Lacour, E E Fullerton, J A Katine, S Mangin

\section{- To cite this version:}

J Cucchiara, Y Henry, D Ravelosona, D Lacour, E E Fullerton, et al.. Telegraph noise due to domain wall motion driven by spin current in perpendicular magnetized nanopillars. Applied Physics Letters, 2009, 94 (10), pp.102503. 10.1063/1.3089569 . hal-02949072

\section{HAL Id: hal-02949072 \\ https://hal.science/hal-02949072}

Submitted on 25 Sep 2020

HAL is a multi-disciplinary open access archive for the deposit and dissemination of scientific research documents, whether they are published or not. The documents may come from teaching and research institutions in France or abroad, or from public or private research centers.
L'archive ouverte pluridisciplinaire HAL, est destinée au dépôt et à la diffusion de documents scientifiques de niveau recherche, publiés ou non, émanant des établissements d'enseignement et de recherche français ou étrangers, des laboratoires publics ou privés. 


\title{
Telegraph noise due to domain wall motion driven by spin current in perpendicular magnetized nanopillars
}

\author{
J. Cucchiara, ${ }^{1}$ Y. Henry, ${ }^{2}$ D. Ravelosona, ${ }^{3}$ D. Lacour, ${ }^{1}$ E. E. Fullerton, ${ }^{4}$ J. A. Katine,${ }^{5}$ and \\ S. Mangin ${ }^{1, a)}$ \\ ${ }_{1}^{1}$ Institut Jean Lamour, UMR CNRS 7198, Nancy Université, UPV Metz, 54506 Vandoeuvre, France \\ ${ }^{2}$ Institut de Physique et Chimie des Matériaux de Strasbourg, UMR CNRS 7504, UDS, France \\ ${ }^{3}$ Institut d'Electronique Fondamentale, UMR CNRS 8622, UPS, 91405 Orsay, France \\ ${ }^{4}$ Center of Magnetic Recording Research, University of California San Diego, California 92093-0401, USA \\ ${ }^{5}$ Hitachi Global Storage Technologies, San Jose Research Center, California 95135, USA
}

(Received 16 January 2009; accepted 5 February 2009; published online 9 March 2009)

\begin{abstract}
Telegraph noise induced by stochastic domain wall nucleation, pinning, and annihilation in the presence of spin transfer torque is observed in nanopillar spin valves with perpendicular anisotropy. By measuring the dwell times corresponding to domain wall pinning and to domain wall nucleation, the telegraph noise is found to be consistent with thermal activation over an effective potential barrier. (C) 2009 American Institute of Physics. [DOI: 10.1063/1.3089569]
\end{abstract}

Recent research has focused on spin-torque switching of devices exhibiting perpendicular magnetic anisotropy. ${ }^{1-4}$ Such systems have the potential for memory application as they provide a pathway in lowering the critical current for reversal $^{5-7}$ and for spin-torque oscillators. ${ }^{2}$ However, in small systems such as nanopillars, the stability of a magnetic state is critical for future applications. For nanopillar spin valves with in-plane anisotropy, in the presence of spin transfer torque telegraph noise (TN) switching has been observed between fully magnetized states: the parallel $(\mathrm{P})$ and the antiparallel (AP) states. ${ }^{8-13}$ In the case of nanopillars exhibiting perpendicular anisotropy no TN effect has been reported, as yet, but has been predicted for certain parts of the currentfield phase diagram. ${ }^{4}$ In addition to the switching between the two uniform states, by the application of both field and current additional intermediate states have been observed in perpendicular anisotropy devices. ${ }^{1,3,14}$ These intermediate states correspond to stable configurations where a domain wall (DW) is pinned in the free layer. Those DWs are found to nucleate and propagate in nanopillars as small as 50 $\times 100 \mathrm{~nm}^{2}$. The study of these configurations and their stability are of great interest to enable applications such as magnetic random access memories or race track memories. ${ }^{15}$

In the present letter, we study the magnetization dynamic of the free layer of nanopillar spin valves with perpendicular anisotropy as both current and magnetic fields are applied perpendicularly to the film plane. Compared to the pillars measured in previous studies ${ }^{1,14}$ the anisotropy of the free layer has been lowered and its area is $100 \times 200 \mathrm{~nm}^{2}$ in order to favor the presence of DW states. The magnetic behavior of the system is monitored through the resistance measurement. As with the previous measurement, we observe transition between the P and AP states with the current and/or field and for certain combinations of field and current we observe several stable intermediate states. In addition, in these samples we observe TN switching that we are able to explain by the stochastic motion of a DW inside the free layer. This behavior is characterized by the dwell time for each DW states which depends strongly on the applied field.

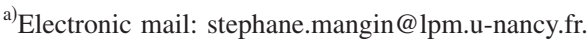

These evolutions can be understood thanks to a Néel-Brown law modified to take into account the spin torque. ${ }^{16}$

The structures studied are made of $\mathrm{Co} / \mathrm{Pt}$ and $\mathrm{Co} / \mathrm{Ni}$ multilayers with perpendicular magnetic anisotropy. They were grown both by coevaporation and dc magnetron sputtering. The magnetic structure consists of a $\operatorname{Pt}(3 \mathrm{~nm}) /[\mathrm{Co}(0.25 \mathrm{~nm}) / \operatorname{Pt}(0.52 \mathrm{~nm})] \times 5 / \mathrm{Co}(0.2 \mathrm{~nm}) /$ $[\mathrm{Ni}(0.6 \mathrm{~nm}) / \mathrm{Co}(0.1 \mathrm{~nm}) /] \times 2 / \mathrm{Co}(0.1 \mathrm{~nm})$ hard reference layer and a $\mathrm{Co}(0.1 \mathrm{~nm}) /[\mathrm{Co}(0.1 \mathrm{~nm}) / \mathrm{Ni}(0.6 \mathrm{~nm} /)] \times 4$ free layer separated by a $4 \mathrm{~nm} \mathrm{Cu}$ spacer layer. Compared to previously published results ${ }^{1,14}$ the free layer is terminated by a $15 \mathrm{~nm} \mathrm{Cu}$ layer instead of a $3 \mathrm{~nm}$ Pt layer. Removing the Pt layer between the free layer and the lead lowers the perpendicular anisotropy of the free layer. The multilayers were then patterned using electron beam lithography and ion etching into nanopillars with an elongated hexagonal shape of $100 \times 200 \mathrm{~nm}^{2}$. The giant magnetoresistance of the system is detected by injecting a dc and measuring its voltage with a nanovoltmeter. This dc current is used both to measure the dc resistance and to inject the current needed for spin transfer torque. The current is defined positive when the electrons flow from the reference layer to the free layer tending to align the two layers in the $\mathrm{P}$ state. The reference layer magnetization switches for an applied field close to $10 \mathrm{kOe}$. Since no fields greater than $1 \mathrm{kOe}$ are applied during the measurements the reference layer is expected to be stable. For all the experiments shown here the reference layer magnetization is pinned along the positive field direction. As presented in previous studies ${ }^{1,14}$ a dipolar stray field $H_{\text {dip }}$ arising from the reference layer is acting on the free layer. $H_{\text {dip }}$ $=350$ Oe for the considered pillars. For clarity purposes figures shall be plotted as a function of the effective field $H_{\text {eff }}$ $=H_{\text {app }}+H_{\text {dip }}$ acting on the free layer.

Figure 1(a) presents the superposition of ten resistance hysteretic curves measured one after the other on the same sample as the effective magnetic field is swept from 900 to -700 Oe and back to 900 Oe with a field sweeping rate of $10 \mathrm{Oe} / \mathrm{s}$ while applying a constant dc current of $I=-2 \mathrm{~mA}$. Those curves show that as the field is swept from positive to negative values, starting from a $\mathrm{P}$ state, the system switches into several intermediate states before reaching the AP state. 

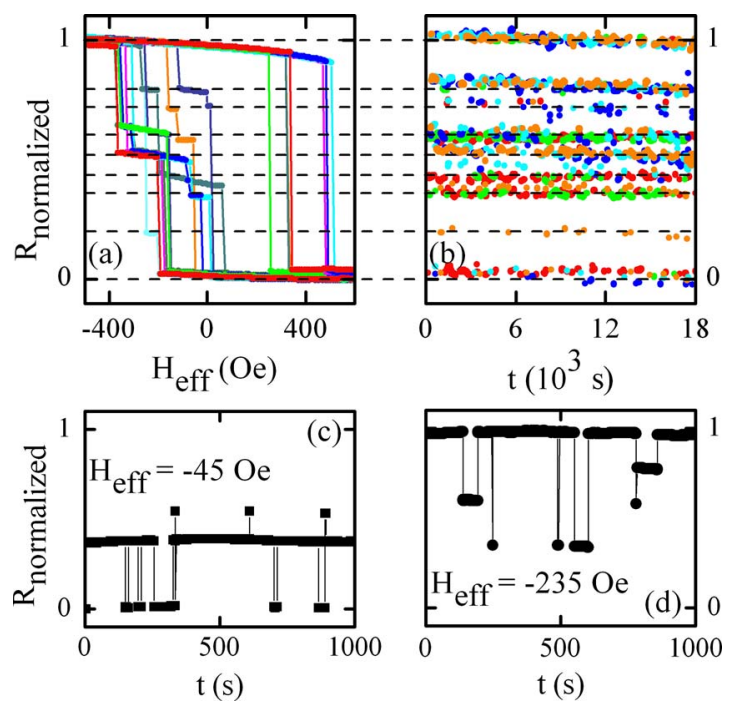

FIG. 1. (Color online) (a) Superposition of ten normalized resistance change $\left(R_{\text {normalized }}\right)$ vs effective field $H_{\text {eff }}$ for an injected current of $-2 \mathrm{~mA}$. (b) Superposition of five normalized resistance changes vs time curves measured with an injected current of $-2 \mathrm{~mA}$ at five different effective magnetic fields from -45 to -235 Oe. The dashed lines highlight that the magnetic states reached during the resistance vs time measurements are the same as the ones obtained during the resistance vs field measurements. (c) and (d) Zoom in the normalized resistance vs time curves measured with an injected current of $-2 \mathrm{~mA}$ and for two different effective fields $H_{\mathrm{eff}}=-45$ Oe and $H_{\text {eff }}=-235$ Oe, respectively.

Up to seven distinct intermediate states could be identified during different field sweeps. However, as the effective field is swept back the system drops directly from the AP to the P state. This symmetry breaking may be understood as follows. Since the $-2 \mathrm{~mA}$ injected current favors an antiparallel alignment, the absolute value of the effective field at the $\mathrm{P}$ to AP state transition is lower than that at the AP to P switching. Consequently, as a low effective field below the intrinsic DW pinning fields of the structure favors the presence of DW states, ${ }^{14}$ this explains why such magnetic configuration is observed on the P to AP branch only. The fact that seven different intermediate levels are well characterized shows that a DW can be pinned at different locations within the free layer. These pinning positions correspond most likely to intrinsic pinning sites present in the film or shape effects and edge roughness introduced by the patterning techniques. ${ }^{14}$ Note also that instead of flat plateaus for each magnetic state, a certain curvature is observed which can be understood by the shape anisotropy and the dipolar field from the hard layer that tend to slightly tilt the magnetization away from the perpendicular direction at the pillar edge when the magnetic field goes to zero.

The large number of pinning locations and the fact that each intermediate state may or may not be visited during any particular field sweep highlights the stochastic dynamic of DW motion. To investigate this effect, the evolution of the nanopillar resistance was measured versus time at a given current and field over $5 \mathrm{~h}$ time periods [Fig. 1(b)]. The current was kept at $-2 \mathrm{~mA}$ and nine different effective fields ranging between -45 and -235 Oe were applied. Those current and field values are the ones for which intermediate states are detected in the hysteresis loops [Fig. 1(a)] and for which TN is observed. Figure 1(b) shows the superposition of five of these measurements whereas Figs. 1(c) and 1(d) show two of them individually. The resistance switches from
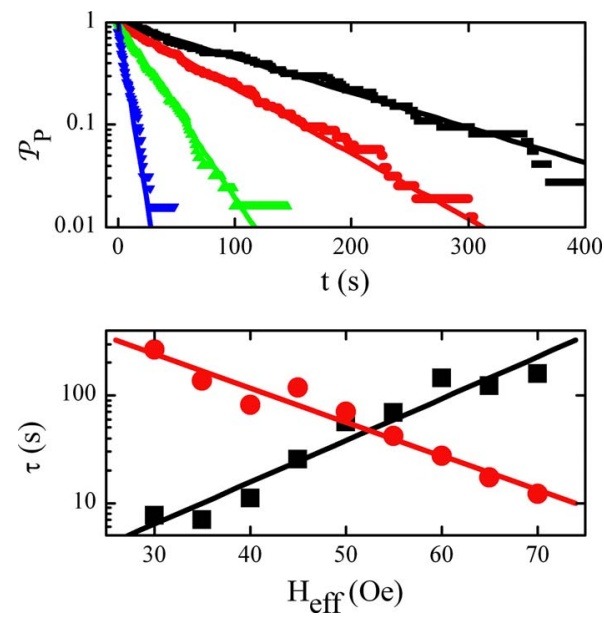

FIG. 2. (Color online) (a) Probability of occupancy of the P state vs time plotted on a semilogarithmic scale for an injected current of $-2.5 \mathrm{~mA}$ and for four different effective magnetic fields: 65 Oe (black $\square$ ), 55 Oe (red $\bigcirc$ ), 45 Oe (green $\mathbf{\Delta}$ ), and 35 Oe (blue $\boldsymbol{\nabla}$ ). (b) Dwell time in the P state (black $\square$ ) and in the domain wall state (red 0 ) plotted on a semilogarithmic scale vs magnetic field for an injected current of $-2.5 \mathrm{~mA}$.

one level to the other and each of the seven intermediate states as well as the P and the AP states shown in Fig. 1(a) may be reached. Only the states observed in the field sweeps are detected in the TN measurements. As a result, these random TN signals may be explained by the creation, the annihilation, and the motion of a DW between different pinning sites activated by thermal fluctuations. ${ }^{17}$ Such behaviors have been observed on the five different pillars that have been studied. The main difference from sample to sample is the number of intermediate states which rises up to seven. This underlines the effect of intrinsic defects randomly distributed in the pillar. ${ }^{14}$

Figures 1(c) and 1(d) show two of the TN scans recorded for $I=-2 \mathrm{~mA}$ and $H_{\mathrm{eff}}=-45$ and $-235 \mathrm{Oe}$, respectively. The resistance jumps back and forth between well-defined positions with a time scale from about a few seconds to a few hundreds of seconds. Those time scales are slightly larger than the ones observed for in-plane anisotropy nanopillars. In that case, $\mathrm{TN}$ is observed between fully magnetized states only with no domain wall involved ${ }^{8-13}$ and the time scales are shown to vary from $1 \mu \mathrm{s}$ to $1 \mathrm{~s}$. For $H_{\mathrm{eff}}=-45$ Oe the system switches mainly between the P state and a DW state whereas for $H_{\mathrm{eff}}=-235$ Oe the system switches between several DW states and the AP state. Both the magnetic states involved and the typical dwell times are tuned by the magnetic field.

In Fig. 2 we show the result for a sample similar in shape and composition as that shown in Fig. 1 but exhibits TN between two magnetic states only: the fully magnetized $\mathrm{P}$ state and one DW state for $I=-2.5 \mathrm{~mA}$. Figure 2(a) shows the probability of staying in the $\mathrm{P}$ state as a function of time after switching in this $\mathrm{P}$ state for various applied fields. This probability is well fitted by a simple exponential curve: $P_{\mathrm{P}}(t)=\exp \left(-t / \tau_{\mathrm{P}}\right)$, where $\tau_{\mathrm{P}}$ is the dwell time in the $\mathrm{P}$ state. For all our samples, the probability of occupancy of a given magnetic state always decays as a simple exponential. This shows that the process studied may be described as a one step thermally activated energy barrier crossing with no memory effect, as it has been seen for others DW depinning processes. ${ }^{18-20}$ 
From those measurements the dwell times in the $\mathrm{P}\left(\tau_{\mathrm{P}}\right)$ and in the DW state $\left(\tau_{D}\right)$ states is deduced for several applied fields at $I=-2.5 \mathrm{~mA}$ [Fig. 2(b)]. $\tau_{\mathrm{P}}$ and $\tau_{D}$ have opposite behaviors. Whereas the DW state is the most stable state, i.e., $\tau_{D}>\tau_{\mathrm{P}}$, for low field values the $P$ state becomes more and more stable, i.e., $\tau_{\mathrm{P}}$ increases for high field values. Both states are equally stable, i.e., $\tau_{D} \sim \tau_{\mathrm{P}}$ around $H_{\mathrm{eff}}=50$ Oe. In Fig. 2(b) the evolution of the dwell times versus field is linear on a semilogarithmic plot. This is consistent with the model proposed in the literature ${ }^{16}$ that considers the NéelBrown formula in the presence of spin torques and gives the following expression for the dwell times,

$$
\tau_{i}=\tau_{0} \exp \left[\frac{E_{i \rightarrow j}(H)\left(1-I / I_{i}^{c}\right)}{k_{B} T}\right],
$$

where $i$ and $j$ are two different magnetic states, $\tau_{0}$ is the attempt time, $E_{i \rightarrow j}$ is the energy barrier from the state $i$ to the state $j, I_{i}^{c}$ is the critical current to reach the state $j$ from the state $i$ at zero temperature, and $k_{B}$ is the Boltzmann constant. Thus we deduce that the evolution of the two energy barriers involved in this TN signal is linear with field. While the system evolves from a P to a DW state this corresponds to a nucleation process that may be describe by an energy barrier given by, ${ }^{21} E_{P \rightarrow I}=2 M_{s} V_{n}\left(H_{n}+H\right)$, where $M_{s}$ is the saturation magnetization, $V_{n}$ is a nucleation volume, and $H_{n}$ a nucleation field. On the other hand, while the system evolves from a DW to a $\mathrm{P}$ state this corresponds to a depinning process and the energy barrier is given by ${ }^{22} E_{I \rightarrow P}=2 M_{s} V_{p}\left(H_{p}+H\right)$, where $V_{p}$ is a characteristic activation volume of the depinning process and $H_{p}$ is the pinning field. Fitting the dwell time evolutions with these two formulas, one could estimate $H_{n} \sim 200$ Oe and $H_{p} \sim 400$ Oe. Supposing that this nucleation energy $2 M_{s} V_{n} H_{n}$ is equal to $K_{a} V_{n}$ the anisotropy energy needed to nucleate and with $M_{s}=500 \mathrm{erg} \mathrm{Oe}^{-1} \mathrm{~cm}^{-3}$ in our sample, we found that $K_{a}$, the anisotropy constant is about $10^{5} \mathrm{erg} / \mathrm{cm}^{3}$ which is close from the value estimated for this sample, ${ }^{5} 1.5 \times 10^{5} \mathrm{erg} / \mathrm{cm}^{3}$. For the pinning field, its value is also close to the value estimated for $200 \mathrm{~nm}$ wide nanowires made from the similar $[\mathrm{Co} / \mathrm{Ni}] /[\mathrm{Co} / \mathrm{Pt}]$ material. ${ }^{23}$ Those quantitative agreements support the idea of thermally assisted magnetization reversal in the presence of a spin transfer torque. This study also shows that in the presence of spin transfer torque one can reach magnetic states that are not accessible using applied fields only by effectively reducing the energy barrier.

In summary, we have observed TN in nanopillars with perpendicular anisotropy as a consequence of thermally activated domain wall nucleation and propagation in presence of the spin transfer torque. The different available magnetization metastable states arise from domain wall pinning induced by the presence of defects.

We thank S. Suire and H. M'Jahed for their help with the experiments and T. Hauet for fruitful discussions. This work was supported in part by the contract ISTRADE of the French National Research Agency (ANR) and by Région Lorraine.

${ }^{1}$ S. Mangin, D. Ravelosona, J. A. Katine, M. J. Carey, B. D. Terris and E. E. Fullerton, Nature Mater. 5, 210 (2006).

${ }^{2}$ D. Houssameddine, U. Ebels, B. Delaet, B. Rodmacq, I. Firastrau, F. Ponthenier, M. Brunet, C. Thirion, J.-P. Michel, L. Prejbeanu-Buda, M.-C. Cyrille, O. Redon, and B. Dieny, Nature Mater. 6, 447 (2007).

${ }^{3}$ T. Seki, S. Mitani, and K. Takanashi, Phys. Rev. B 77, 214414 (2008).

${ }^{4}$ R. Zhu and P. B. Visscher, J. Appl. Phys. 103, 07 A722 (2008).

${ }^{5}$ S. Mangin, Y. Henry, D. Ravelosona, J. A. Katine, and E. E. Fullerton, Appl. Phys. Lett. 94, 012502 (2009).

${ }^{6}$ M. Nakayama, T. Kai, N. Shimomura, M. Amano, E. Kitagawa, T. Nagase, M. Yoshikawa, T. Kishi, S. Ikegawa and H. Yoda, J. Appl. Phys. 103, 07A710 (2008).

${ }^{7}$ T. Seki, S. Mitani, K. Yakushiji, and K. Takanashi, Appl. Phys. Lett. 89, 172504 (2006).

${ }^{8}$ E. B. Myers, F. J. Albert, J. C. Sankey, E. Bonet, R. A. Buhrman, and D. C. Ralph, Phys. Rev. Lett. 89, 196801 (2002).

${ }^{9}$ S. Urazhdin, N. O. Birge, W. P. Pratt, Jr., and J. Bass, Phys. Rev. Lett. 91, 146803 (2003).

${ }^{10}$ S. Urazhdin, H. Kurt, W. P. Pratt, Jr., and J. Bass, Appl. Phys. Lett. 83, 114 (2003).

${ }^{11}$ A. Fabian, C. Terrier, S. Serrano Guisan, X. Hoffer, M. Dubey, L. Gravier J.-Ph. Ansermet, and J.-E. Wegrowe, Phys. Rev. Lett. 91, 257209 (2003).

${ }^{12}$ I. N. Krivorotov, N. C. Emley, A. G. F. Garcia, J. C. Sankey, S. I. Kiselev, D. C. Ralph, and R. A. Buhrman, Phys. Rev. Lett. 93, 166603 (2004).

${ }^{13}$ W. L. Lim, N. Anthony, A. Higgins, and S. Urazhdin, Appl. Phys. Lett. 92, 172501 (2008).

${ }^{14}$ D. Ravelosona, S. Mangin, Y. Lemaho, J. A. Katine, B. D. Terris, and E. E. Fullerton, Phys. Rev. Lett. 96, 186604 (2006).

${ }^{15}$ M. Hayashi, L. Thomas, R. Moriya, C. Rettner, and S. S. Parkin, Science 320, 209 (2008).

${ }^{16} \mathrm{Z}$. Li and S. Zhang, Phys. Rev. B 69, 134416 (2004).

${ }^{17}$ P. Gaunt, J. Appl. Phys. 59, 4129 (1986).

${ }^{18}$ S. Mangin, A. Sulpice, G. Marchal, C. Bellouard, W. Wernsdorfer, and B. Barbara, Phys. Rev. B 60, 1204 (1999).

${ }^{19}$ S. Mangin, G. Marchal, W. Wernsdorfer, A. Sulpice, K. Hasselbach, D. Mailly, and B. Barbara, Europhys. Lett. 39, 675 (1997).

${ }^{20}$ J. P. Attané, D. Ravelosona, A. Marty, Y. Samson, and C. Chappert, Phys. Rev. Lett. 96, 147204 (2006)

${ }^{21}$ J.-P. Jamet, S. Lemerle, P. Meyer, J. Ferré, B. Bartenlian, N. Bardou, C. Chappert, P. Veillet, F. Rousseaux, D. Decanini, and H. Launois, Phys. Rev. B 57, 14320 (1998).

${ }^{22}$ J. Ferré, in Spin Dynamics in Confined Magnetic Structures I, edited by B. Hillebrands and K. Ounadjela (Springer, New York, 2002), Vol. 83, p. 127

${ }^{23}$ D. Ravelosona, S. Mangin, J. A. Katine, E. E. Fullerton, and B. D. Terris, Appl. Phys. Lett. 90, 072508 (2007). 